Gabriele Scheidt · Der Kolportagebuchhandel (1869-1905) 


\section{Gabriele Scheidt}

\section{Der Kolportagebuchhandel (1869-1905)}

Eine systemtheoretische Rekonstruktion

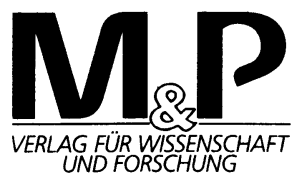


Die Deutsche Bibliothek - CIP-Einheitsaufnahme

\section{Scheidt, Gabriele:}

Der Kolportagebuchhandel (1869 - 1905); eine systemtheoretische

Rekonstruktion / Garbiele Scheidt. - Stuttgart : M und P, Verl.

für Wiss. und Forschung, 1994

Zugl.: München, Univ., Diss., 1992

ISBN 978-3-476-45046-3

\section{ISBN 978-3-476-45046-3}

ISBN 978-3-476-04203-3 (eBook)

DOI 10.1007/978-3-476-04203-3

Dieses Werk ist einschließlich aller seiner Teile geschützt. Jede Verwertung außerhalb der engen Grenzen des Urheberrechtsgesetzes ist ohne Zustimmung des Verlages unzulässig und strafbar. Das gilt insbesondere für Vervielfältigungen, Übersetzungen, Mikroverfilmungen und Einspeicherung in elektronischen Systemen.

M \& $\mathbf{P}$ Verlag für Wissenschaft und Forschung ein Verlag der J. B.Metzlerschen Verlagsbuchhandlung und Carl Emst Poeschel Verlag GmbH in Stuttgart

C 1994 Springer-Verlag GmbH Deutschland

Ursprünglich erschienen bei J. B. Metzlersche Verlagbuchhandlung und Carl Ernst Poeschel Verlag GmbH in Stuttgart 1994 
»Plüsch - der Stoff, in dem sich besonders leicht Spuren abdrücken.« WALTER BENJAMIN, Das Passagen-Werk 
Dem Andenken an Henning Zwirner 


\section{Vorbemerkung}

Die vorliegende Arbeit wurde im Wintersemester 1992/93 von der Philosophischen Fakultät für Sprach- und Literaturwissenschaft II der Ludwig-Maximilians-Universität München als Dissertation angenommen; sie wurde für die Druckfassung nur unwesentlich verändert. Die Arbeit wurde von Prof. Dr. Jäger angeregt, wofür ich ihm Dank schulde; das Koreferat hat freundlicherweise Frau Prof. Dr. von Heydebrandt übernommen.

Ohne ein Stipendium der Studienstiftung des Deutschen Volkes hätte ich das Projekt niemals beginnen, geschweige denn zu einem Abschluß bringen können. Ich bin der Organisation für ihre unbürokratische und tatkräftige Förderung zu herzlichem Dank verpflichtet.

Forschungsprojekte gedeihen nicht in einem Vakuum: ein langjähriger Arbeitskreis, der sich der Anwendung systemtheoretischer Konzepte auf die Literaturwissenschaft verschrieben hatte, gab der Untersuchung wichtige Anregungen.

Der Kolportageroman ist aus dem kollektiven Gedächtnis der Bibliotheken und buchhandelsgeschichtlichen Institutionen verschwunden: daß er dennoch überlebt hat, ist in beträchtlichem Maße Günter Kosch zu verdanken, der in jahrelanger Arbeit die verstreuten Exemplare gesammelt und bibliographisch erfaßt hat. Von ihm habe ich viele wertvolle Hinweise erhalten. Diese Arbeit ist unter denkbar widrigen Umständen entstanden; sie hat Opfer gekostet, die allein die Betroffenen zu ermessen wissen. Daß sie dennoch abgeschlossen wurde, habe ich der Unterstützung meiner Freunde und dem Beistand meiner Familie zu verdanken. Ganz besonderer Dank gilt Otto Neudeck, der mein Tun mit kritischem Engagement und aufmunterndem Gespräch begleitet hat. 


\section{Inhaltsverzeichnis}

Einleitung

\section{A Funktion}

Forschungsüberblick und Problemkonstruktion

1 Im Dickicht der Begriffe:

Die literatursoziologische Trivialliteraturforschung

2 Der ,triviale Text ${ }^{\text {' }} \mathrm{zw}$ wischen Anerkennungsbemühung und Aufklärungsinteresse: Bedürfnisfunktionalismus und Ideologiekritik

3 Die strukturfunktionalistische Gegenstandskonstitution eine systemtheoretische Reformulierung.............................44

3.1 Das Kausalprinzip als Modus der Behandlung von Komplexität ......48

3.1.1 Komplexitätsreduktion und ihre Folgen: Der Zwang zur Asymmetrisierung im Bedürfnisfunktionalismus .....................52

3.1.2 Kausalannahmen als Modus der Komplexitätssteigerung in der Ideologiekritik .58

3.2 Der Strukturfunktionalismus im Selbstkontakt ........................61

3.2.1 Die Selbstbeobachtung der bedürfnisfunktionalistischen Richtung ....64

3.2.2 Die Selbstapplikation des ideologiekritischen Ansatzes ..............67

3.3 Zusammenfassung .............................................71

\section{B Reflexion}

Der Ort der Trivialliteratur im Literatursystem eine systemtheoretische Modellbildung ....

1 Das Literatursystem als Beispiel einer nicht gelungenen

Systemdifferenzierung ............................................... 78

1.1 Die gesellschaftliche Präsenz des Literatursystems ...................89

1.2 Literatur und das Problem der Wertekonversion .......................93 
2 Literarischer Leistungsaustausch

2.1 Die Organisation der Ressourcen

2.2 Die Brauchbarkeit literarischer Produkte:

Ein selbstgeschaffenes Paradox

2.3 Imagination versus Erkenntnis oder das Selbstbefriedigungsverbot im Literatursystem

3 Der Kolportageroman als Beispiel leistungsorientierter Literatur -

Versuch einer Textsortenbestimmung

\section{Leistung}

Die Rekonstruktion des Kolportagebuchhandels zwischen 1869 und 1905

1 Bedingungen der Ausdifferenzierung des Kolportagebuchhandels...138

1.1 Rechtliche Voraussetzungen ........................................141

1.2 Die Entwicklung zum selbständigen Vertriebsmodus .................147

2 Anfänge der Selbstorganisation ..................................... 156

2.1 Räumliche Distinktion: Berlin versus Leipzig ........................159

2.2 Sachliche Differenzierung: Zentralverein versus Verband .............162

3 Der Zentralverein deutscher Kolportagebuchhändler

Selbstbeschreibung und Interessenvertretung.

3.1 Der Kolportagebuchhändler als ,Kaufmann des Buchhandels ‘ und ,Pionnier der Cultur

3.2 Der Antrag Gröber und Genossen zur Änderung der Gewerbeordnung (1893)

4 Die Verkehrsordnung des Zentralvereins: Regulierung systeminterner Relationen und interne Differenzierung ...............176

4.1 ,Unberechtigte Concurrenz' oder ,Collegenfabrikaton' '...............182

4.2 Der Deutsche Colportage-Buchhändler-Verein ,Palm` ................187

4.3 ,Viribus unitis‘: Aktiengesellschaft und Kommissionshaus ...........193

5 Der Weichert-Verlag als Prototyp eines ,Gesamt-Verlagsbetriebes ‘..199 
6 Der Warenvertrieb ..................................................212

6.1 Prämie und Prospekt: Programmierung und Konversion der Werte ...222

6.2 Die Änderung der Gewerbeordnung von 1883.....................232

6.3 Im Zeichen des Sammelmaterials:

Die Fiktionalisierung der Kommunikation

7 Der Kolportageroman ...............................................249

7.1 ,Das Buch als Ware':

Überlegungen zur Brauchbarkeit des Kolportageromans .............255

7.2 Die verlorene Werkherrschaft des Kolportageroman-Autors.........266

7.3 Der Kolportageroman als Imaginationsinstrument:

Der ,Kampf gegen die Schundliteratur'

\section{Anhang}

Anmerkungen zur Quellenlage von Kolportagebuchhandel und

Kolportageroman

Die Fachzeitschriften des Kolportagebuchhandels.

Ein chronologischer Überblick ........................................298

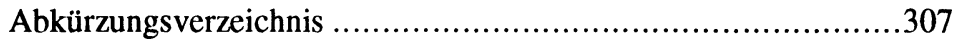

\section{Literaturverzeichnis}

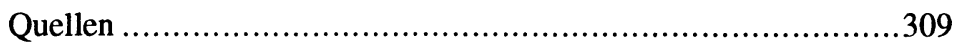

Forschungsliteratur .............................................. 328 